\title{
GLOBAL WEAK SOLUTIONS TO 1D COMPRESSIBLE ISENTROPIC NAVIER-STOKES EQUATIONS WITH DENSITY-DEPENDENT VISCOSITY*
}

\author{
SONG JIANG ${ }^{\dagger}, Z_{\text {ZHOUPING XIN } \ddagger \text { AND PING ZHANG }}$
}

Key words. Compressible Navier-Stokes equations, isentropic gas, freee boundary, weak solutions

AMS subject classifications. $35 \mathrm{Q} 10$

1. Introduction. We establish the global existence and uniqueness of weak solutions to the Navier-Stokes equations for a one-dimensional isentropic viscous gas with a jump to the vacuum initially when the viscosity depends on the density:

$$
\left\{\begin{array}{l}
\rho_{\tau}+(\rho u)_{\xi}=0 \\
(\rho u)_{\tau}+\left(\rho u^{2}+P(\rho)\right)_{\xi}=\left(\mu(\rho) u_{\xi}\right)_{\xi},
\end{array}\right.
$$

in $\tau>0, a(\tau)<\xi<b(\tau)$, where $\rho, u$ and $P(\rho)$ are the density, the velocity and the pressure, respectively, $\mu(\rho) \geq 0$ is the viscosity coefficient, $a(\tau)$ and $b(\tau)$ are the free boundaries, i.e. the interface of the gas and the vacuum:

$$
\left\{\begin{array}{l}
\frac{d}{d \tau} a(\tau)=u(a(\tau), \tau), \frac{d}{d \tau} b(\tau)=u(b(\tau), \tau), \\
\left(-P(\rho)+\mu(\rho) u_{\xi}\right)(a(\tau), \tau)=0, \quad\left(-P(\rho)+\mu(\rho) u_{\xi}\right)(b(\tau), \tau)=0 .
\end{array}\right.
$$

Due to the strong degeneracy at vacuum, both Euler and Navier-Stokes systems for compressible fluids (in which the viscosity is independent of density) behave singularly $[7,10,16]$. In particular, the classical one-dimensional isentropic Navier-Stokes system picks up unphysical solutions for two gases initially separated by vacuum states $[7,10]$. To overcome this difficulty, Liu, Xin and Yang in [10] introduced the modified Navier-Stokes system (1.1) in which the viscosity coefficient depends on the density. It is shown in [10] that at least locally in time, the system (1.1) yields the physically relevant solution. As remarked by Liu, Xin and Yang in [10], the model is also motivated by the physical consideration that in the derivation of the compressible Navier-Stokes equations from the Boltzmann equations, the viscosity is not constant and depends on the temperature. For isentrpoic flow, this dependence is translated into the dependence of the viscosity on the density.

For simplicity we consider in this paper

$$
\left\{\begin{array}{l}
P(\rho)=A \rho^{\gamma} \\
\mu(\rho)=B \rho^{\alpha}
\end{array}\right.
$$

where $\gamma>1, A>0, B>0, \alpha>0$ are constants.

* Received June 17, 2005; accepted for publication November 25, 2005.

$\dagger$ IMS, The Chinese University of Hong Kong, Shatin, New Territories, Hong Kong. Current Address: Institute of Applied Physics and Computational Mathematics, P.O. Box 8009, Beijing 100088, China (jiang@mail.iapcm.ac.cn).

$\ddagger$ IMS, The Chinese University of Hong Kong, Shatin, New Territories, Hong Kong; and Center of Nonlinear Studies, Northwest University, China (zpxin@ims.cuhk.edu.hk).

$\S$ IMS, The Chinese University of Hong Kong, Shatin, New Territories, Hong Kong. Current Address: Academy of Mathematics and System Sciences, Chinese Academy of Sciences, Beijing 100080, China (zp@amss.ac.cn). 
To study the free boundary (1.1), (1.2), it is convenient to convert the free boundaries to the fixed boundaries by using Lagrangian coordinates. We introduce the coordinate transformation:

$$
x=\int_{a(\tau)}^{\xi} \rho(y, \tau) d y, \quad t=\tau
$$

then the free boundaries $a(\tau)$ and $b(\tau)$ become

$$
x=0 \text { and } x=\int_{a(\tau)}^{b(\tau)} \rho(y, \tau) d y=\int_{a}^{b} \rho(y, 0) d y
$$

with $a:=a(0)$ and $b:=b(0)$, where $\int_{a}^{b} \rho(y, 0) d y$ is the total mass initially. Without loss of generality we assume $\int_{a}^{b} \rho(y, 0) d y=1$. Hence, in Lagrangian coordinates, the free boundary problem (1.1), (1.2) becomes

$$
\begin{gathered}
\rho_{t}+\rho^{2} u_{x}=0, \\
u_{t}+A\left[\rho^{\gamma}\right]_{x}=B\left[\rho^{1+\alpha} u_{x}\right]_{x}, \quad 0<x<1, t>0,
\end{gathered}
$$

with the boundary conditions

$$
A \rho^{\gamma}=B \rho^{1+\alpha} u_{x}, \quad \text { at } x=0 \text { and } x=1, \quad t \geq 0 .
$$

We impose the following initial conditions

$$
(\rho(x, 0), u(x, 0))=\left(\rho_{0}(x), u_{0}(x)\right), \quad x \in[0,1] .
$$

As pointed out in [14], physicists claim that the viscosity of a gas is proportional to the square root of the temperature (e.g. see [4], also see [5, 9]). The temperature is of order $\rho^{\gamma-1}$, provided that the pressure $P$ is proportional to the product of the density and the temperature, i.e. the perfect fluid. In this case we have $\alpha=(\gamma-1) / 2$.

From the boundary conditions (1.6) we easily obtain that for $\gamma>\alpha, \rho(0, t), \rho(1, t)$ $=O\left(t^{-1 /(\gamma-\alpha)}\right)$ for $t$ large. Hence, the density grows with $t$, thus causing the viscosity (the stabilization mechanism) to decrease to zero, a solution may not exist globally in time. We shall show that if $\mu(\rho)$ does not decrease to zero too rapidly, i.e. if $\alpha$ is not large, then a weak solution of (1.4)-(1.7) still exists globally in time.

When the viscosity is constant, the free boundary problems for one-dimensional compressible Navier-Stokes equations were investigated in $[3,1,2,11]$ (also see [15, $6,16]$ for the Cauchy problem) and among others, where the global existence of weak solutions was proved. When the viscosity does depend on the density, a local existence theorem was obtained by Makino, Liu, Xin and Yang [12, 10], where the initial density was assumed to be connected to vacuum with discontinuities. Jiang in [8] studied the Navier-Stokes equations for a one-dimensional heat-conducting gas and proved the global existence of smooth solutions provided that $0<\alpha<1 / 4$ in (1.3). Using techniques similar to those in [8] to derive a priori estimates and the finite difference method, Okada, Matušu-Nečasová and Makino [14] obtained the existence of global weak solutions in the case of isentropic flow for $0<\alpha<1 / 3$. Their result was improved recently to the case $0<\alpha<1 / 2$ by Yang, Yao and Zhu [17]. In $[14,17]$ the initial data are required to satisfy $\rho_{0}, \partial_{x} u_{0} \in \operatorname{Lip}[0,1]$.

In this paper we prove the global existence of solutions to (1.4)-(1.7) under the conditions $\rho_{0} \in W^{1, p}(0,1), u_{0} \in L^{p}(0,1)$ for some $p$ and $0<\alpha<1$. Furthermore, we establish the uniqueness provided $u_{0} \in H^{1}(0,1)$. Our result improves those in [14, 
16]. The improvement is twofold: More general pressure laws can be dealt with here, for example for the perfect fluid $(\alpha=(\gamma-1) / 2) 1<\gamma<2$ is assumed in $[14,16]$ while $1<\gamma<3$, which is for most gases, is allowed in the present paper; less regularity of the initial data is required.

Now before stating the main result, we introduce the notation used throughout this paper: Let $m \geq 0$ be an non-negative integer and let $1 \leq p \leq \infty$. By $W^{m, p}$ we denote the usual Sobolev space defined over $(0,1)$ with norm $\|\cdot\|_{W^{m, p}} ; H^{m} \equiv W^{m, 2}$ with norm $\|\cdot\|_{H^{m}}, L^{p} \equiv W^{0, p}$ with norm $\|\cdot\|_{L^{p}} . L^{p}(I, B)$ resp. $\|\cdot\|_{L^{p}(I, B)}$ denotes the space of all strongly measurable, $p$ th-power integrable (essentially bounded if $p=\infty$ ) functions from $I$ to $B$ resp. its norm, $I \subset \mathbb{R}$ an interval, $B$ a Banach space. Let $\beta \in(0,1), C^{\beta}[0,1]$ denotes the Banach space of functions on $[0,1]$ which are uniformly Hölder continuous with exponent $\beta$ and $C^{\beta, \beta / 2}\left(Q_{T}\right)$ for the Banach space of functions on $Q_{T}:=[0,1] \times[0, T]$ which are uniformly Hölder continuous with exponents $\beta$ in $x$ and $\beta / 2$ in $t$.

The main result of this paper reads:

Theorem 1.1. Assume $\gamma>1$ and $0<\alpha<1$ in (***, $\inf _{[0,1]} \rho_{0}>0, \rho_{0} \in W^{1,2 n}$, $u_{0} \in L^{2 n}$ for some $n \in \mathbb{N}$ satisfying $n(2 n-1) /\left(2 n^{2}+2 n-1\right)>\alpha$. Then the initialboundary problem (1.4)-(1.7) possesses a global weak solution $(\rho, u)$ in the sense that for any $T>0$,

$$
\begin{aligned}
& \rho \in L^{\infty}\left([0, T], W^{1,2 n}\right), \rho_{t} \in L^{2}\left([0, T], L^{2}\right), u \in L^{\infty}\left([0, T], L^{2 n}\right) \cap L^{2}\left([0, T], H^{1}\right), \\
& \rho(x, t) \geq C \text { on }[0,1] \times[0, T]
\end{aligned}
$$

for some positive constant $C=C\left(\left\|\rho_{0}\right\|_{W^{1,2 n}},\left\|u_{0}\right\|_{L^{2 n}}, \inf _{[0,1]} \rho_{0}, T\right)$, and the following equations hold:

$$
\begin{gathered}
\rho_{t}+\rho^{2} u_{x}=0, \quad \rho(x, 0)=\rho_{0}(x) \quad \text { for a.e. } x \in(0,1) \text { and any } t \geq 0, \\
\int_{0}^{\infty} \int_{0}^{1}\left\{u \phi_{t}+\left(A \rho^{\gamma}-B \rho^{\alpha+1} u_{x}\right) \phi_{x}\right\} d x d t+\int_{0}^{1} u_{0}(x) \phi(x, 0) d x=0
\end{gathered}
$$

for any test function $\phi(x, t) \in C_{0}^{\infty}(Q)$ with $Q:=\{(x, t) \mid 0 \leq x \leq 1, t \geq 0\}$.

Moreover, if, in addition, $u_{0} \in H^{1}$, then $u$ satisfies the additional estimates:

$$
u \in L^{\infty}\left([0, T], H^{1}\right) \cap L^{2}\left([0, T], H^{2}\right), u_{t} \in L^{2}\left([0, T], L^{2}\right),
$$

and furthermore, this weak solution is unique in the class:

$$
\begin{aligned}
& \rho \in L^{\infty}\left([0, T], H^{1}\right), \rho_{t} \in L^{2}\left([0, T], L^{2}\right), u \in L^{\infty}\left([0, T], L^{2}\right) \cap L^{2}\left([0, T], H^{1}\right), \\
& \rho(x, t)>0 \text { on }[0,1] \times[0, T] .
\end{aligned}
$$

The proof of Theorem 1.1 is based on a priori estimates for the approximate solutions of (1.4)-(1.7) and a limit procedure. To derive the a priori estimates, the crucial step is to obtain lower and upper bounds of the density, that is, if the initial density has no vacuum and concentration of mass on $[0,1]$, then the same should be true for the density for all $t>0$. By exploiting the high integrability of $u,\left(\rho^{\alpha}\right)_{x}$ (i.e. $u,\left(\rho^{\alpha}\right)_{x} \in L^{\infty}\left([0, T], L^{2 n}\right), u^{n-1} u_{x} \in L^{2}\left([0, T], L^{2}\right)$ for any $\left.n \in \mathbb{N}\right)$ and the energy conservation, we obtain thus the boundedness of the density from below and above.

The paper is organized as follows: In Section 2 we derive the a priori estimates and prove the existence by constructing an approximate solution and taking to the limit. The uniqueness is given in Section 3. 
2. Proof of the existence. In this section we first derive the a priori estimates for $(\rho, u)$, then we construct the approximate solutions by mollifying the initial data and obtain the global existence by taking to the limit. Throughout this section the same letter $C$ (sometimes used as $C(X, Y, \cdots)$ to emphasize the dependence of $C$ on $X, Y, \cdots)$ will denote various positive constants which may depend on $\left\|\rho_{0}\right\|_{W^{1,2 n}}$, $\left\|u_{0}\right\|_{L^{2 n}}, \inf _{[0,1]} \rho_{0}$ and $T$ with $n$ being the same as in Theorem 1.1.

Let $(\rho(x, t), u(x, t))$ be a solution of $(1.4)-(1.7)$ on $[0, T]$ satisfying

$$
\begin{aligned}
& \rho, \rho_{x}, \rho_{t}, \rho_{t x}, u, u_{x}, u_{t}, u_{x x} \in C^{\beta, \beta / 2}\left(Q_{T}\right) \quad \text { for some } 0<\beta<1, \\
& \rho(x, t)>0 \text { on } Q_{T} .
\end{aligned}
$$

To show the a priori estimates we begin with the following lemma:

LEMMA 2.1. We have

$$
\begin{gathered}
\int_{0}^{1}\left(\frac{u^{2}(x, t)}{2}+\frac{A \rho^{\gamma-1}(x, t)}{\gamma-1}\right) d x+B \int_{0}^{t} \int_{0}^{1} \rho^{1+\alpha} u_{x}^{2} d x d s \\
=\int_{0}^{1}\left(\frac{u_{0}^{2}}{2}+\frac{A \rho_{0}^{\gamma-1}}{\gamma-1}\right) d x \quad \forall t \in[0, T],
\end{gathered}
$$

and

$$
\rho(x, t) \leq C \quad \forall x \in[0,1], t \in[0, T]
$$

and for any $n \in \mathbb{N}$,

$$
\int_{0}^{1} u^{2 n}(x, t) d x+n(2 n-1) \int_{0}^{t} \int_{0}^{1} u^{2 n-2} \rho^{1+\alpha} u_{x}^{2} d x d s \leq C \quad \forall t \in[0, T],
$$

where $C=C\left(\left\|\rho_{0}\right\|_{L^{\infty}},\left\|u_{0}\right\|_{L^{2}}\right)$ in (2.3) and $C=C\left(\left\|\rho_{0}\right\|_{L^{\infty}},\left\|u_{0}\right\|_{L^{p}}, T, n\right)$ in (2.4).

Proof. Multiplying (1.5) by $u$, integrating the resulting equation over $(0,1) \times(0, t)$, integrating by parts, using the boundary conditions (1.6) and the equation (1.4), we obtain (2.2). (2.3) can be shown easily by using the equation (1.4) and (2.2) while (2.4) follows from the multiplication of (1.5) with $2 n u^{2 n-1}$ in $L^{2}$ and integration by parts, their proof can be found in [17]. (see Lemmas 2.4 and 2.6 in [17].)

LEMma 2.2. There is a positive constant $C=C\left(\left\|\rho_{0}\right\|_{W^{1,2 n}},\left\|u_{0}\right\|_{L^{2 n}}, T\right)$, such that

$$
\int_{0}^{1}\left(\partial_{x} \rho^{\alpha}\right)^{2 n}(x, t) d x \leq C, \quad t \in[0, T]
$$

Proof. We may write (1.4) in the form:

$$
\frac{1}{\alpha} \partial_{t} \rho^{\alpha}+\rho^{1+\alpha} u_{x}=0 .
$$

Thus, substituting (2.5) into (1.5) and integrating over $[0, t]$, we obtain

$$
u(x, t)-u_{0}(x)+A \int_{0}^{t}\left(\rho^{\gamma}\right)_{x}(x, s) d s=\frac{B}{\alpha}\left(\partial_{x} \rho_{0}^{\alpha}-\partial_{x} \rho^{\alpha}\right) .
$$


We multiply (2.6) by $\left(\partial_{x} \rho^{\alpha}\right)^{2 n-1}(n \in \mathbb{N})$ and integrate over $(0,1)$ with respect to $x$ to get

$$
\begin{aligned}
\int_{0}^{1}\left(\partial_{x} \rho^{\alpha}\right)^{2 n} d x= & \int_{0}^{1}\left(\partial_{x} \rho^{\alpha}\right)^{2 n-1} \partial_{x} \rho_{0}^{\alpha} d x \\
& +\frac{\alpha}{B} \int_{0}^{1}\left\{\left(u-u_{0}\right)+A \int_{0}^{t}\left(\rho^{\gamma}\right)_{x} d s\right\}\left(\partial_{x} \rho^{\alpha}\right)^{2 n-1} d x \\
\leq & C\left(\int_{0}^{1}\left(\partial_{x} \rho^{\alpha}\right)^{2 n} d x\right)^{(2 n-1) / 2 n} \\
& \cdot\left\{\left\|\partial_{x} \rho_{0}^{\alpha}\right\|_{L^{2 n}}+\left\|u-u_{0}\right\|_{L^{2 n}}+\int_{0}^{t}\left\|\partial_{x} \rho^{\gamma}\right\|_{L^{2 n}} d s\right\} .
\end{aligned}
$$

Using (2.4), Young's inequality $a b \leq a^{p} / p+b^{q} / q(1 / p+1 / q=1, p, q>1, a, b \geq 0)$, we get from (2.7) that there is a positive constant $C$ depending on $\left\|\rho_{0}\right\|_{W^{1,2 n}},\left\|u_{0}\right\|_{L^{2 n}}$ and $T$, such that

$$
\int_{0}^{1}\left(\partial_{x} \rho^{\alpha}\right)^{2 n}(x, t) d x \leq \frac{1}{2} \int_{0}^{1}\left(\partial_{x} \rho^{\alpha}\right)^{2 n}(x, t) d x+C \int_{0}^{t} \int_{0}^{1}\left(\partial_{x} \rho^{\gamma}\right)^{2 n} d x d s+C,
$$

whence,

$$
\int_{0}^{1}\left(\partial_{x} \rho^{\alpha}\right)^{2 n}(x, t) d x \leq C+C \int_{0}^{t} \max _{[0,1]}\left(\rho^{\gamma-\alpha}\right)^{2 n} \int_{0}^{1}\left(\partial_{x} \rho^{\alpha}\right)^{2 n} d x d s .
$$

Applying Gronwall's inequality to (2.8) and making use of (2.3), we obtain the lemma. This completes the proof.

Lemma 2.3. Let $n \in \mathbb{N}$ be fixed such that $\left(2 n^{2}-n\right) /\left(2 n^{2}+2 n-1\right)>\alpha$. Then there is a positive constant $C=C\left(\left\|u_{0}\right\|_{L^{2 n}},\left\|\rho_{0}\right\|_{W^{1,2 n}}, \inf _{[0,1]} \rho_{0}, T\right)$, such that

$$
\rho(x, t) \geq C \quad \forall x \in[0,1], t \in[0, T] .
$$

Proof. Set

$$
v(x, t)=\frac{1}{\rho(x, t)}, \quad \text { and } \quad V(t)=\max _{[0,1] \times[0, t]} v(x, s) .
$$

It then follows from Lemma 2.2 that

$$
\begin{aligned}
v(x, t)-v(0, t) & =\int_{0}^{x} \partial_{x} v d x \leq \int_{0}^{1}\left|\partial_{x} \rho\right| v^{2} d x \\
& =\frac{1}{\alpha} \int_{0}^{1}\left|\partial_{x} \rho^{\alpha}\right| v^{1+\alpha} d x \\
& \leq \frac{1}{\alpha}\left(\int_{0}^{1}\left(\partial_{x} \rho^{\alpha}\right)^{2 n} d x\right)^{1 / 2 n}\left(\int_{0}^{1} v^{(1+\alpha) q} d x\right)^{1 / q} \\
& \leq C\left(\int_{0}^{1} v(x, t) d x\right)^{1 / q}\left(\max _{[0,1]} v(\cdot, t)\right)^{[(1+\alpha) q-1] / q},
\end{aligned}
$$

where $q=2 n /(2 n-1)$. 
The equation (1.4) can be written as $v_{t}=u_{x}$. Integrating this over $[0,1] \times[0, t]$ and using Young's inequality, we deduce that

$$
\begin{aligned}
\int_{0}^{1} v(x, t) d x & =\int_{0}^{t}(u(1, s)-u(0, s)) d s+\int_{0}^{1} v(x, 0) d x \\
& \leq 2 \int_{0}^{t} \max _{[0,1]}|u(\cdot, s)| d s+C \\
& \leq C\left(\int_{0}^{t} \max _{[0,1]}|u(\cdot, s)|^{n} d s\right)^{1 / n}+C .
\end{aligned}
$$

The integral term on the right-hand side of (2.10) can be bounded as follows, using Sobolev's imbedding theorem and (2.4).

$$
\begin{aligned}
\int_{0}^{t}\left\|u^{n}(s)\right\|_{L^{\infty}} d s & \leq C \int_{0}^{t}\left\|u^{n}(s)\right\|_{W^{1,1}} d s \\
& \leq C \int_{0}^{t}\left\|u^{2 n}(s)\right\|_{L^{1}}^{1 / 2} d s+C \int_{0}^{t} \int_{0}^{1}|u|^{n-1}\left|u_{x}\right| d x d s \\
& \leq C+C\left(\int_{0}^{t} \int_{0}^{1} u^{2 n-2} u_{x}^{2} \rho^{1+\alpha} d x d s\right)^{1 / 2}\left(\int_{0}^{t} \int_{0}^{1} v^{(1+\alpha)} d x d s\right)^{1 / 2} \\
& \leq C+C\left(\int_{0}^{t} \int_{0}^{1} v^{(1+\alpha)} d x d s\right)^{1 / 2} .
\end{aligned}
$$

Hence, inserting the above estimate into (2.10), we conclude that

$$
\int_{0}^{1} v(x, t) d x \leq C+C\left(\int_{0}^{t} \int_{0}^{1} v^{(1+\alpha)} d x d s\right)^{1 /(2 n)} .
$$

We may write $(1.4)$ in the form: $\left[\rho^{-(1-\alpha) / 2}\right]_{t}=\frac{(1-\alpha)}{2} \rho^{(1+\alpha) / 2} u_{x}$. Integration of this equation over $[0, x] \times[0, t]$ yields

$$
v^{(1-\alpha) / 2}(x, t)=\rho_{0}^{-(1-\alpha) / 2}(x)+\frac{(1-\alpha)}{2} \int_{0}^{t} \rho^{(1+\alpha) / 2} u_{x} d s .
$$

Therefore, integrating the above identity over $(0,1)$ and using $(2.2)$, we arrive at

$$
\begin{aligned}
\int_{0}^{1} v^{(1-\alpha)}(x, t) d x & \leq 2 \int_{0}^{1} \rho_{0}^{-(1-\alpha)} d x+C \int_{0}^{1}\left(\int_{0}^{t} \rho^{(1+\alpha) / 2}\left|u_{x}\right| d s\right)^{2} d x \\
& \leq 2 \int_{0}^{1} \rho_{0}^{-(1-\alpha)} d x+C \int_{0}^{t} \int_{0}^{1} \rho^{1+\alpha} u_{x}^{2} d x d s \\
& \leq C\left(\left\|\rho_{0}\right\|_{L^{\infty}},\left\|u_{0}\right\|_{L^{2}}, \inf _{[0,1]} \rho_{0}, T\right) .
\end{aligned}
$$

Combining (2.11) with (2.12), we see that

$$
\begin{aligned}
\int_{0}^{1} v(x, t) d x & \leq C+C\left(\int_{0}^{t} \int_{0}^{1} v^{2 \alpha} v^{1-\alpha} d x d s\right)^{1 /(2 n)} \\
& \leq C+C V^{\alpha / n}(t) .
\end{aligned}
$$

Now, substitution of (2.13) into (2.9) gives us

$$
v(x, t) \leq v(0, t)+C V(T)^{\alpha /(n q)+[(1+\alpha) q-1] / q}
$$


for all $x \in[0,1], t \in[0, T]$. By the boundary conditions (1.6) we have

$$
-\frac{A}{B} \rho^{\gamma-\alpha+1}(0, t)=\rho_{t}(0, t)
$$

which yields

$$
v(0, t)=v(0,0)\left(\frac{(\gamma-\alpha) A}{B} \rho_{0}^{\gamma-\alpha} t+1\right)^{1 /(\gamma-\alpha)} \leq C(T), \quad t \in[0, T] .
$$

Inserting (2.15) into (2.14) and taking into account that

$$
\frac{\alpha}{n q}+\frac{(1+\alpha) q-1}{q}<1 \quad \text { with } q=\frac{2 n}{2 n-1}
$$

because of $\alpha<n(2 n-1) /\left(2 n^{2}+2 n-1\right)$, we obtain

$$
V(T) \leq C\left(\left\|\rho_{0}\right\|_{W^{1,2 n}},\left\|u_{0}\right\|_{L^{2 n}}, \inf _{[0,1]} \rho_{0}, T\right) \quad \forall x \in[0,1], t \in[0, T] .
$$

This completes the proof of the lemma.

Having established the a priori estimates Lemmas 2.1-2.3, we are now in a position to prove the existence of weak solutions.

Proof of the existence. We denote by $j_{\epsilon}(x)$ the Friedrichs mollifier. Let $\psi(x) \in$ $C_{0}^{\infty}(\mathbb{R})$ satisfy $\psi(x)=1$ when $|x| \leq 1 / 2$ and $\psi(x)=0$ when $|x| \geq 1$, and define $\psi_{\epsilon}(x):=\psi(x / \epsilon)$. For simplicity we still denote by $\left(\rho_{0}, u_{0}\right)$ the extension of $\left(\rho_{0}, u_{0}\right)$ in $\mathbb{R}$, i.e.

$$
\rho_{0}(x):=\left\{\begin{array}{c}
\rho_{0}(1), x \in(1, \infty), \\
\rho_{0}(x), x \in[0,1], \\
\rho_{0}(0), x \in(-\infty, 0),
\end{array} \quad u_{0}(x):=\left\{\begin{array}{l}
u_{0}(x), x \in[0,1], \\
0, \quad \text { otherwise }
\end{array}\right.\right.
$$

We define the approximate initial data to $\rho_{0}, u_{0}$ :

$$
\begin{aligned}
\rho_{0}^{\epsilon}(x):= & \left(\rho_{0} * j_{\epsilon}\right)(x) \\
u_{0}^{\epsilon}(x):= & \left(u_{0} * j_{\epsilon}\right)(x)\left\{1-\psi_{\epsilon}(x)-\psi_{\epsilon}(1-x)\right\} \\
& +\left(u_{0} * j_{\epsilon}\right)(0) \psi_{\epsilon}(x)+\left(u_{0} * j_{\epsilon}\right)(1) \psi_{\epsilon}(1-x) \\
& +\frac{A}{B}\left(\rho_{0}^{\epsilon}\right)^{\gamma-\alpha-1}(0) \int_{0}^{x} \psi_{\epsilon}(y) d y-\frac{A}{B}\left(\rho_{0}^{\epsilon}\right)^{\gamma-\alpha-1}(1) \int_{x}^{1} \psi_{\epsilon}(1-y) d y .
\end{aligned}
$$

Then, $\rho_{0}^{\epsilon} \in C^{1+\beta}[0,1], u_{0}^{\epsilon} \in C^{2+\beta}[0,1]$ for any $0<\beta<1$, and $\rho_{0}^{\epsilon}$ and $u_{0}^{\epsilon}$ are compatible with the boundary conditions (1.6). Recalling the definition of the Friedrichs mollifier, we have

$$
\begin{aligned}
\left|\left(u_{0} * j_{\epsilon}\right)(0)\right|^{2 n} \int_{0}^{1} \psi_{\epsilon}^{2 n}(x) d x & \leq C \epsilon\left(\int_{0}^{\epsilon} u_{0}(x) j_{\epsilon}(x) d x\right)^{2 n} \\
& \leq C \epsilon \int_{0}^{\epsilon} u_{0}^{2 n} d x\left(\int_{0}^{\epsilon} j_{\epsilon}^{2 n /(2 n-1)}(x) d x\right)^{2 n-1} \\
& \leq C \int_{0}^{\epsilon} u_{0}^{2 n}(x) d x \rightarrow 0 \text { as } \epsilon \rightarrow 0 .
\end{aligned}
$$

In the same manner, $\left|\left(u_{0} * j_{\epsilon}\right)(1)\right|^{2 n} \int_{0}^{1} \psi_{\epsilon}^{2 n}(1-x) d x \rightarrow 0$. Therefore, recalling the definition of $u_{0}^{\epsilon}(x)$ we easily see that as $\epsilon \rightarrow 0$,

$$
\rho_{0}^{\epsilon} \rightarrow \rho_{0} \quad \text { in } W^{1,2 n}, \quad u_{0}^{\epsilon} \rightarrow u_{0} \quad \text { in } L^{2 n} .
$$


Now, consider the initial boundary value problem (1.4)-(1.7) with the initial data $\left(\rho_{0}, u_{0}\right)$ replaced by $\left(\rho_{0}^{\epsilon}, u_{0}^{\epsilon}\right)$. For this problem we can apply the standard argument (the energy estimates and the contraction mapping theorem) to obtain the existence of a unique local solution $\left(\rho^{\epsilon}, u^{\epsilon}\right)$ with $\rho^{\epsilon}, \rho_{x}^{\epsilon}, \rho_{t}^{\epsilon}, \rho_{t x}^{\epsilon}, u^{\epsilon}, u_{x}^{\epsilon}, u_{t}^{\epsilon}, u_{x x}^{\epsilon} \in C^{\beta, \beta / 2}([0,1] \times$ $\left.\left[0, T^{*}\right]\right)$ for some $T^{*}>0$. In view of Lemmas 2.1-2.3 and (2.17) we see that $\rho^{\epsilon}$ is pointwise bounded from below and above, $\left(u^{\epsilon}\right)^{n}$ and $\rho_{x}^{\epsilon}$ are bounded in $L^{\infty}\left([0, T], L^{2}\right)$, and $u_{x}^{\epsilon}$ is bounded in $L^{2}\left((0, T), L^{2}\right)$ for any $T>0$. Furthermore, we can differentiate the equations (1.4)-(1.5) and apply the energy method to derive bounds of high-order derivative of $\left(\rho^{\epsilon}, u^{\epsilon}\right)$, then we can apply the Schauder theory for linear parabolic equations to conclude that the $C^{\beta, \beta / 2}\left(Q_{T}\right)$-norm of $\rho^{\epsilon}, \rho_{x}^{\epsilon}, \rho_{t}^{\epsilon}, \rho_{t x}^{\epsilon}, u^{\epsilon}, u_{x}^{\epsilon}, u_{t}^{\epsilon}$ and $u_{x x}^{\epsilon}$ is a priori bounded. Therefore, we can continue the local solution globally in time and obtain that there exists a unique global solution $\left(\rho^{\epsilon}, u^{\epsilon}\right)$ of $(1.4)-(1.7)$ with $\left(\rho_{0}, u_{0}\right)$ replaced by $\left(\rho_{0}^{\epsilon}, u_{0}^{\epsilon}\right)$, such that for any $T>0,(2.1)$ for $\left(\rho^{\epsilon}, u^{\epsilon}\right)$ holds. (see e.g. the book by Antontsev, Kazhikhov and Monakhov [3] concerning compressible viscous heatconducting fluids.) Moreover, by virtue of Lemmas 2.1-2.3, (2.17) and the equation (1.5) and we have the following uniform in $\epsilon$ estimates:

$$
\begin{aligned}
& \int_{0}^{1}\left(u^{\epsilon}\right)^{2 n}(x, t) d x+\int_{0}^{1}\left(\rho^{\epsilon}\right)_{x}^{2 n}(x, t) d x \leq C, \quad t \in[0, T], n \in \mathbb{N}, \\
& C^{-1} \leq \rho^{\epsilon}(x, t) \leq C \quad \forall x \in[0,1], t \in[0, T], \\
& \int_{0}^{T} \int_{0}^{1}\left\{\left(u^{\epsilon}\right)_{x}^{2}+\left(\rho_{t}^{\epsilon}\right)^{2}\right\}(x, s) d x d s \leq C,
\end{aligned}
$$

where $C$ is a positive constant which depends on $\left\|\rho_{0}\right\|_{W^{1,2 n}},\left\|u_{0}\right\|_{L^{2 n}}, \inf _{[0,1]} \rho_{0}, T$ and $n$, but not on $\epsilon$. Thus, we can extract a subsequence of $\left(\rho^{\epsilon}, u^{\epsilon}\right)$, still denoted by $\left(\rho^{\epsilon}, u^{\epsilon}\right)$, such that as $\epsilon \rightarrow 0$,

$$
\begin{aligned}
& u^{\epsilon} \rightarrow u \text { weak- } * \text { in } L^{\infty}\left([0, T], L^{2 n}\right), \\
& \rho^{\epsilon} \rightarrow \rho \text { weak-* in } L^{\infty}\left([0, T], W^{1,2 n}\right), \\
& \left(\rho_{t}^{\epsilon}, u_{x}^{\epsilon}\right) \rightarrow\left(\rho_{t}, u_{x}\right) \text { weakly in } L^{2}\left([0, T], L^{2}\right) .
\end{aligned}
$$

Next we show that $(\rho, u)$ obtained in (2.19) is a weak solution of (1.4)-(1.7). By Sobolev's embedding theorem $W^{1,2 n}(0,1) \hookrightarrow C^{1-1 /(2 n)}[0,1]$, we have for any $x_{1}, x_{2} \in$ $(0,1), t \in[0, T]$ that

$$
\left|\rho^{\epsilon}\left(x_{1}, t\right)-\rho^{\epsilon}\left(x_{2}, t\right)\right| \leq C\left|x_{1}-x_{2}\right|^{1-1 /(2 n)} .
$$

On the other hand, it follows from Lions-Aubin's lemma and (2.18) that for any $\delta>0$, there is some constant $C_{\delta}>0$, such that for any $t_{1}, t_{2} \in[0, T]$,

$$
\begin{aligned}
& \left\|\rho^{\epsilon}\left(t_{1}\right)-\rho^{\epsilon}\left(t_{2}\right)\right\|_{L^{\infty}} \\
& \quad \leq \delta\left\|\rho^{\epsilon}\left(t_{1}\right)-\rho^{\epsilon}\left(t_{2}\right)\right\|_{W^{1, p}}+C_{\delta}\left\|\rho^{\epsilon}\left(t_{1}\right)-\rho^{\epsilon}\left(t_{2}\right)\right\|_{L^{2}} \\
& \quad \leq 2 \delta\left\|\rho^{\epsilon}(t)\right\|_{W^{1, p}}+C_{\delta}\left|t_{1}-t_{2}\right|^{1 / 2}\left\|\rho_{t}^{\epsilon}\right\|_{L^{2}\left([0, T], L^{2}\right)} \\
& \quad \leq C \delta+C_{\delta}\left|t_{1}-t_{2}\right|^{1 / 2} .
\end{aligned}
$$

Thus, (2.20)-(2.21) together with the triangle inequality show that $\left\{\rho^{\epsilon}(x, t)\right\}$ is equicontinuous on $[0,1] \times[0, T]$. Hence, by Arzéla-Ascoli's theorem and a diagonal process for $t$, we can extract a subsequence of $\left\{\rho^{\epsilon}\right\}$, still denoted by $\left\{\rho^{\epsilon}\right\}$, such that

$$
\rho^{\epsilon}(x, t) \rightarrow \rho(x, t) \text { strongly in } C^{0}\left(Q_{T}\right) .
$$


Moreover, by virtue of (2.21),

$$
\rho \in C^{\frac{1}{2}}\left([0, T], L^{2}\right)
$$

Now, we multiply (1.5) by $\phi \in C_{0}^{\infty}(Q), Q=\{(t, x) \mid t \geq 0,0 \leq x \leq 1\}$ and integrate over $(0, T) \times(0,1)$, then we integrate by parts with respect to $t$ and $x$ and take to the limit as $\epsilon \rightarrow 0$. If we use (2.17)-(2.19), (2.22) and (2.23), we easily see that $(\rho, u)$ obtained in $(2.19)$ satisfies $(1.8)-(1.9)$. Hence, $(\rho, u)$ is a weak solution. The proof of the existence is complete.

3. Uniqueness. We first prove the regularity of the weak solution constructed in Section 2 for smoother data. Then we show the uniqueness of the weak solutions under the additional regularity assumption upon $u_{0}$. Throughout this section, we will assume that $u_{0} \in H^{1}([0,1])$, and the same letter $C$ (sometimes used as $C(X, Y, \cdots)$ to emphasize the dependence of $C$ on $X, Y, \cdots)$ will denote various positive constants which may depend on $\left\|\rho_{0}\right\|_{W^{1,2 n}},\left\|u_{0}\right\|_{H^{1}}, \inf _{[0,1]} \rho_{0}$ and $T$ with $n$ being the same as in Theorem 1.1.

Lemma 3.1. Let $(\rho, u)$ be the weak solution of (1.4)-(1.7) established in Section 2. Then $(\rho, u)$ satisfies (1.10) and

$$
\begin{array}{r}
\left\|u_{t}\right\|_{L^{2}\left(Q_{T}\right)}+\left\|u_{x x}\right\|_{L^{2}\left(Q_{T}\right)}+\left\|u_{x}\right\|_{L^{\infty}\left([0, T], L^{2}\right)} \\
\leq C\left(\left\|u_{0}\right\|_{H^{1}},\left\|\rho_{0}\right\|_{W^{1,2 n}}, \inf _{[0,1]} \rho_{0}, T\right)
\end{array}
$$

where $n$ is the same as in Theorem 1.1.

Proof. Let $\left(\rho^{\epsilon}, u^{\epsilon}\right)$ denote the global smooth solution of (1.4)-(1.7) with the initial data $\left(\rho_{0}^{\epsilon}, u_{0}^{\epsilon}\right)$ given in Section 2, which satifies (2.1). First we show that $\partial_{x} u_{0}^{\epsilon} \rightarrow \partial_{x} u_{0}$ in $L^{2}$. In fact, consider the term $\left\{\left(u_{0} * j_{\epsilon}\right)(x)-\left\{\left(u_{0} * j_{\epsilon}\right)(0)\right\} \partial_{x} \psi_{\epsilon}(x)\right.$ in $\partial_{x} u_{0}^{\epsilon}$. We have

$$
\begin{aligned}
\int_{0}^{1}\left\{\left(u_{0} * j_{\epsilon}\right)(x)-\left(u_{0} * j_{\epsilon}\right)(0)\right\}^{2}\left(\partial_{x} \psi_{\epsilon}\right)^{2} d x & \leq \frac{C}{\epsilon^{2}} \int_{0}^{\epsilon}\left\{\left(u_{0} * j_{\epsilon}\right)(x)-\left(u_{0} * j_{\epsilon}\right)(0)\right\}^{2} d x \\
& \leq \frac{C}{\epsilon} \max _{[0, \epsilon]}\left\{\left(u_{0} * j_{\epsilon}\right)(\cdot)-\left(u_{0} * j_{\epsilon}\right)(0)\right\}^{2} \\
& =\frac{C}{\epsilon}\left(\int_{0}^{\epsilon}\left|\partial_{x}\left(u_{0} * j_{\epsilon}\right)\right| d x\right)^{2} \\
& \leq C \int_{0}^{\epsilon}\left|\partial_{x}\left(u_{0} * j_{\epsilon}\right)\right|^{2} d x \rightarrow 0 .
\end{aligned}
$$

In the same manner, $\int_{0}^{1}\left\{\left(u_{0} * j_{\epsilon}\right)(x)-\left(u_{0} * j_{\epsilon}\right)(1)\right\}^{2}\left[\partial_{x} \psi_{\epsilon}(1-x)\right]^{2} d x \rightarrow 0$. Hence, we easily deduce that

$$
\partial_{x} u_{0}^{\epsilon} \rightarrow \partial_{x} u_{0} \quad \text { in } L^{2}
$$

For simplicity, from now on until the end of the proof of this lemma we will drop out the supscript $\epsilon$.

Multiplying the equation $(1.5)$ by $u_{t}$ in $L^{2}([0,1] \times[0, T])$ and taking into account 
the boundary conditions (1.6), we integrate by parts to get

$$
\begin{aligned}
& \int_{0}^{t} \int_{0}^{1} u_{t}^{2} d x d s+\frac{B}{2} \int_{0}^{1} \rho^{1+\alpha} u_{x}^{2} d x=\frac{B}{2} \int_{0}^{1} \rho_{0}^{1+\alpha}\left(u_{0}\right)_{x}^{2} d x \\
& \quad+A \int_{0}^{1}\left(\rho^{\gamma} u_{x}\right)(x, t) d x-A \int_{0}^{1} \rho_{0}^{\gamma} \partial_{x} u_{0} d x \\
& \quad+A \gamma \int_{0}^{t} \int_{0}^{1} \rho^{\gamma+1} u_{x}^{2} d x d s-\frac{B(1+\alpha)}{2} \int_{0}^{t} \int_{0}^{1} \rho^{2+\alpha} u_{x}^{3} d x d s
\end{aligned}
$$

where we have also used the equation (1.4). It is easy to see that by virtue of Lemmas $2.1-2.3$, the inequality $2|a b| \leq \delta a^{2}+\delta^{-1} b^{2},(\delta>0),(2.17)$ and (3.2), the identity (3.3) implies

$$
\int_{0}^{1} u_{x}^{2} d x+\int_{0}^{t} \int_{0}^{1} u_{t}^{2} d x d s \leq C+C \int_{0}^{t} \int_{0}^{1}\left|\rho^{1+\alpha} u_{x}^{3}\right| d x d s, \quad t \in[0, T] .
$$

The last term on the right-hand side of (3.4) can be estimated as follows, using (2.3), (2.2), and Lemma 2.3, the boundary conditions (1.6) and the equation (1.4).

$$
\begin{aligned}
C & \int_{0}^{t} \int_{0}^{1}\left|\rho^{1+\alpha} u_{x}^{3}\right| d x d s \leq C \int_{0}^{t} \max _{[0,1]}\left|\left(\rho^{1+\alpha} u_{x}\right)(\cdot, s)\right| \int_{0}^{1} u_{x}^{2} d x d s \\
& \leq C \int_{0}^{t} \max _{[0,1]}\left|\left(B \rho^{1+\alpha} u_{x}-A \rho^{\gamma}\right)(\cdot, s)\right| \int_{0}^{1} u_{x}^{2} d x d s+C \int_{0}^{t} \int_{0}^{1} u_{x}^{2} d x d s \\
& \leq C \int_{0}^{t} \int_{0}^{1}\left|\left(B \rho^{1+\alpha} u_{x}-A \rho^{\gamma}\right)_{x}(x, s)\right| d x \int_{0}^{1} u_{x}^{2} d x d s+C \\
& =C \int_{0}^{t} \int_{0}^{1}\left|u_{t}\right| d x \int_{0}^{1} u_{x}^{2} d x d s+C \\
& \leq \frac{1}{2} \int_{0}^{t} \int_{0}^{1} u_{t}^{2} d x d s+C \int_{0}^{t}\left(\int_{0}^{1} u_{x}^{2} d x\right)^{2} d s+C .
\end{aligned}
$$

Inserting the above estimate into (3.4), we obtain

$$
\int_{0}^{1} u_{x}^{2}(x, t) d x+\int_{0}^{t} \int_{0}^{1} u_{t}^{2} d x d s \leq C+C \int_{0}^{t}\left\|u_{x}(s)\right\|_{L^{2}}^{2} \int_{0}^{1} u_{x}^{2} d x d s
$$

for $t \in[0, T]$, If we apply Gronwall's inequality to (3.5), and use (2.2) and Lemma 2.3, we infer that

$$
\int_{0}^{1} u_{x}^{2}(x, t) d x+\int_{0}^{t} \int_{0}^{1} u_{t}^{2} d x d s \leq C .
$$

From the equation (1.5) we see by Lemmas 2.1-2.3 and (3.6) that $\left\|u_{x x}\right\|_{L^{2}\left(Q_{T}\right)}^{2}$ is also bounded from above by a positive constant. Hence, the weak solution obtained in (2.19) satisfies (1.10). Moreover, by the lower semicontinuity properties of the weak-* and weak topology, (3.1) holds. This completes the proof the lemma.

Now, we are able to prove the uniqueness in Theorem 1.1

Proof of the uniqueness. Let $\left(\rho_{1}(x, t), u_{1}(x, t)\right)$ be an arbitrary weak solution to (1.4)-(1.7) which is in the class $(1.11)$. Let $\left(\rho_{2}(x, t), u_{2}(x, t)\right)$ be the weak solution constructed in Section 2, which has the additional regularity (1.10) as proved in Lemma 3.1. We will show that these two solutions have to coincide. For convenience, we set 
$v_{i}(x, t)=\frac{1}{\rho_{i}(x, t)}$ for $i=1,2$. First we note that by virtue of $(1.4),\left(\rho_{1}, u_{1}\right)$ and $\left(\rho_{2}, u_{2}\right)$ satisfy

$$
\rho_{i}^{1+\alpha} \partial_{x} u_{i}=-\frac{1}{\alpha} \partial_{t} \rho_{i}^{\alpha}, \quad \partial_{x} u_{i}=\partial_{t} v_{i}, \quad i=1,2 .
$$

Then, from (1.5) and (3.7), we get

$$
\left(u_{1}-u_{2}\right)_{t}+A\left(\rho_{1}^{\gamma}-\rho_{2}^{\gamma}\right)_{x}=B\left(\rho_{1}^{1+\alpha} u_{1 x}-\rho_{2}^{1+\alpha} u_{2 x}\right)_{x} .
$$

Multiplying the above equation by $u_{1}-u_{2}$ in $L^{2}(0,1)$, integrating by parts and using the boundary conditions (1.6) and (3.7), we find that

$$
\begin{aligned}
\frac{1}{2} \frac{d}{d t} & \int_{0}^{1}\left(u_{1}-u_{2}\right)^{2} d x \\
& =A \int_{0}^{1}\left(v_{1}-v_{2}\right)_{t}\left(v_{1}^{-\gamma}-v_{2}^{-\gamma}\right) d x-B \int_{0}^{1}\left(u_{1 x}-u_{2 x}\right)^{2}\left(\rho_{1}^{1+\alpha}\right) d x \\
& -B \int_{0}^{1}\left(u_{1}-u_{2}\right)_{x}\left(v_{1}^{-(1+\alpha)}-v_{2}^{-(1+\alpha)}\right) u_{2 x} d x \\
\leq & -C_{0} \int_{0}^{1}\left(u_{1 x}-u_{2 x}\right)^{2} d x+C_{1} \int_{0}^{1}\left(u_{2 x}\right)^{2}\left(v_{1}-v_{2}\right)^{2} d x \\
- & \frac{d}{d t} \int_{0}^{1} a(x, t)\left(v_{1}-v_{2}\right)^{2} d x+\int_{0}^{1} a_{t}(x, t)\left(v_{1}-v_{2}\right)^{2} d x,
\end{aligned}
$$

where $C_{0}$ and $C_{1}$ are positive constants depending only on the upper and lower bounds of $\rho_{1}$ and $\rho_{2}$, and $a(x, t)$ is defined as:

$$
a(x, t):=\frac{A \gamma}{2} \int_{0}^{1}\left(v_{2}+\tau\left(v_{1}-v_{2}\right)\right)^{-(\gamma+1)} d \tau,
$$

which has a positive lower bound on $[0,1] \times[0, T]$. Since

$$
\left|a_{t}(x, t)\right| \leq C\left(\left|v_{2 t}\right|+\left|v_{1 t}-v_{2 t}\right|\right)
$$

with a positive constant $C$ depending only on the lower and upper bounds of $\rho_{1}$ and $\rho_{2}$, as can be checked directly, one has that

$$
\begin{aligned}
\int_{0}^{1} a_{t}(x, t)\left(v_{1}-v_{2}\right)^{2} d x \\
\quad \leq \frac{C_{0}}{2} \int_{0}^{1}\left(v_{1 t}-v_{2 t}\right)^{2} d x+C \int_{0}^{1}\left(1+\left|v_{2 t}\right|\right)\left(v_{1}-v_{2}\right)^{2} d x .
\end{aligned}
$$

Then, using this in (3.8) and integrating the resulting estimate over $(0, t)$, we deduce that

$$
\begin{aligned}
& \frac{1}{2} \int_{0}^{1}\left(u_{1}-u_{2}\right)^{2} d x+\int_{0}^{1} a(x, t)\left(v_{1}-v_{2}\right)^{2} d x+\frac{C_{0}}{2} \int_{0}^{t} \int_{0}^{1}\left(u_{1 x}-u_{2 x}\right)^{2} d x d s \\
& \quad \leq \int_{0}^{t} \int_{0}^{1}\left(1+\left|u_{2 x}\right|\right)^{2}\left(v_{1}-v_{2}\right)^{2} d x d s
\end{aligned}
$$


which, taking into account $a(x, t) \geq C>0$, gives

$$
\begin{array}{rl}
\int_{0}^{1}\left(u_{1}-u_{2}\right)^{2} & d x+\int_{0}^{1}\left(v_{1}(x, t)-v_{2}(x, t)\right)^{2} d x \\
\leq C \int_{0}^{t} \int_{0}^{1}\left(1+\left|u_{2 x}\right|\right)^{2}\left(v_{1}(x, s)-v_{2}(x, s)\right)^{2} d x d s .
\end{array}
$$

Since $u_{2 x} \in L^{2}\left([0, T], H^{1}\right)$ we find by Sobolev's imbedding theorem that $\int_{0}^{t} \|(1+$ $\left.\left|u_{2 x}\right|\right)^{2}(s) \|_{L^{\infty}} d s<\infty$. Hence, an application of Gronwall's inequality to (3.9) yields immediately that

$$
v_{1}(x, t)=v_{2}(x, t), \quad u_{1}(x, t)=u_{2}(x, t) \quad \text { a.e. }(x, t) \in[0,1] \times[0, T] .
$$

The proof of the uniqueness is complete.

Acknowledgement. This research is supported in part by Zheng-Ge Ru Foundation. This paper was done when Jiang and Zhang were visiting the IMS, the Chinese University of Hong Kong. The hospitality of the institute is greatly appreciated. The research of Jiang and Zhang is supported also by the CNSF, the Climbing Project of Nonlinear Science, the Ministry of Education and the CAEP of China. The research of Xin is supported in part by Hong Kong RGC Earmarked Research Grants and a research grant at Center of Nonlinear Studies at Northwest University (China).

\section{REFERENCES}

[1] A.A. Amosov, A.A. Zlotnik, Global generalized solutions of the equations of the one-dimensional motion of a viscous heat-conducting gas, Soviet Math. Dokl, 38 (1989), pp. 1-5.

[2] Solvability "in the large" of a system of equations of the one-dimensional motion of an inhomogeneous viscous heat-conducting gas, Math. Notes, 52 (1992), pp. 753-763.

[3] S.N. Antontsev, A.V. Kazhikhov, V.N. Monakhov, Boundary Value Problems in Mechanics of Nonhomogeneous Fluids, North-Holland, Amsterdam, New York, 1990.

[4] R. Balian, From microphysics to macrophysics, Texts and Monographs in Physics, SpringerVerlag, Berlin, Heidelberg, 1982.

[5] H. Grad, Asymptotic theory of the Boltzmann equation. II, in Rarefied Gas Dynamics 1, J. Laurmann, eds., Academic Press, New York, 1963, pp. 26-59.

[6] D. Hoff, Global solutions of the equations of one-dimensional, compresssible flow with large data and forces, and with deffering end states, Z. Angew. Math. Phys., 49 (1998), pp. 774785.

[7] D. Hoff, D. Serre, The failure of continuous dependence on initial data for the Navier-Stokes equations of compressible flow, SIAM J. Math. Anal., 51 (1991), pp. 887-898.

[8] S. JIANG, Global smooth solutions of the equations of a viscous, heat-conducting, one-dimensional gas with density-dependent viscosity, Math. Nachr., 190 (1998), pp. 169-183.

[9] S. Kawashima, A. Matsumura, T. Nishida, On the fluid-dynamical approximation to the Boltzmann equation at the level of the Navier-Stokes equation, Commun. Math. Phys., 70 (1979), pp. 97-124.

[10] T.-P. LiU, Z. Xin, T. Yang, Vacuum states for compressible flow, Discrete Continuous Dyn. Sys., 4 (1998), pp. 1-32.

[11] T. LUO, Z. XIN, T. YANG, Interface behavior of compressible Navier-Stokes equations with vacuum, SIAM J. Math. Anal., 31 (2000), pp. 1175-1191.

[12] T. Makino, On a local existence theorem for the evolution equations of gaseous stars, in Patterns and Wave-qualitative Analysis of Nonlinear Differential Equations, North-Holland, 1986, pp. 459-479.

[13] M. OKADA, Free boundary value problems for the equation of one-dimensional motion of viscous gas, Japan J. Indust. Appl. Math., 6 (1989), pp. 161-177. 
[14] M. Okada, S̆. Matuš $\dot{u}$-NeC̆asová, T. Makino, Free boundary problem for the equation of one-dimensional motion of compressible gas with density-dependent viscosity, Ann. Univ. Ferrara Sez. VII (N.S.), 48 (2002), pp. 1-20.

[15] D. Serre, Sur l'équation monodimensionnelle d'un fluids visqueux, compressible et conducteur de chaleur, C.R. Acad. Sci. Paris Sér I, 303 (1986), pp. 703-706.

[16] Z. XIN, Blow-up of smooth solutions to the compressible Navier-Stokes equations with compact density, Comm. Pure Appl. Math., 51 (1998), pp. 229-240.

[17] T. YAng, Z.-A. YAO, C.-J. ZHU, Compressible Navier-Stokes equations with density-dependent viscosity and vacuum, Comm. Partial Differential Equations, 26 (2001), pp. 965-981. 
S. JIANG, Z. XIN AND P. ZHANG 